\title{
Quality of Life after Bilateral and Contralateral Prophylactic Mastectomy with Implant Reconstruction
}

\author{
Rüdiger Klapdor Christina Weiß Elna Kuehnle Fabian Kohls Julia von Ehr \\ Anja Philippeit Ursula Hille-Betz \\ Department of Gynecology and Obstetrics, Hannover Medical School, Hannover, Germany
}

\section{Keywords}

Prophylactic mastectomy · Hereditary breast cancer ·

Contralateral mastectomy $\cdot$ BRCA

\begin{abstract}
Background: Prophylactic mastectomy is an effective approach to breast cancer risk reduction in patients at high risk. Further studies using standardized measures for quality of life are needed to better understand the effect of prophylactic mastectomy on individual patients and, thereby, allow for better patient counseling and selection. Methods: In this prospective study patients undergoing bilateral mastectomy were asked to complete the BREAST-Q questionnaire before and 1 year after surgery. All patients underwent bilateral mastectomy with implant-based breast reconstruction. Patient- and surgery-related information was collected in a database. Results: In total, 48 patients underwent bilateral skin-sparing mastectomy. Of these, 29 (60.4\%) suffered from breast cancer. A 2-stage reconstruction with intermediate expander implantation was conducted in 19 (39.6\%) patients. All patients completed the BREAST-Q questionnaire. The domain "psychosocial well-being" was significantly improved from a mean score of 74.98 preoperatively to a postoperative score of $81.56(p=0.021)$. In contrast, the domain "physical well-being" dropped -8.38 points on average to a postoperative score of $74.96(p<0.001)$. Interestingly, pa-
\end{abstract}

tients with the lowest preoperative score in the domain "satisfaction with breast" showed the greatest increase after surgery $(50.31$ vs. $67.25, p<0.001)$. On the contrary, patients with the highest preoperative values experienced the strongest decrease in satisfaction ( 91.60 vs. $75.27, p=0.012$ ). Conclusion: Implant-based prophylactic mastectomy leads to good quality-of-life results in patients at high risk for breast cancer. Especially, patients with a low preoperative satisfaction with their breasts have a significantly higher chance of experiencing substantial improvements in their quality of life.

(c) 2020 S. Karger AG, Basel

\section{Introduction}

Prophylactic mastectomy (PM) is the therapeutic option offering the strongest breast cancer risk reduction for women at high risk such as those with BRCA1 or BRCA2 mutations or a strong family history of breast cancer [1]. Women with BRCA1 or BRCA2 mutations have a lifetime risk of developing breast cancer of $40-80 \%$ [2]. Furthermore, patients with BRCA1/BRCA2 mutations already diagnosed with breast cancer have an elevated risk of contralateral breast cancer ranging between 20 and $80 \%$. According to a recent meta-analysis, bilateral PM (BPM) as well as contralateral PM (CPM) for patients al- 
ready diagnosed with breast cancer offer a strong risk reduction of 90 and $93 \%$, respectively [1]. Consequently, there has been an increasing demand for these procedures over the last years. However, these surgeries might be associated with perioperative complications as well as severe affection of the body image, which might impact the patient's psychosocial, physical, and sexual well-being [2-8]. Besides extensive experience in the field of PM, reliable data of multiple studies is mandatory for comprehensive counseling of patients. However, most studies analyzing satisfaction and quality of life after BPM or CPM have only evaluated postoperative results. A few studies have recorded preoperative values which allow for sufficient evaluation [9-11]. The publication by McCarthy et al. [8] reports a particularly important study. They assessed the patients' satisfaction and quality of life using the BREAST-Q questionnaire in 204 patients with BPM (implant-based and autologous reconstruction). The BREAST-Q questionnaire is a patient related outcome instrument on satisfaction and quality of life designed for patients undergoing breast related surgery [12]. McCarthy et al. [8] were able to show that the domains "psychosocial well-being" and "satisfaction with breast" were significantly higher postoperatively compared to preoperative results. However, "physical well-being/chest and upper body" was significantly worse at 1 year follow-up. More studies, specifically focused on implant-based surgery, evaluating pre- and postoperative satisfaction with breast cancer specific questionnaires are urgently needed to better understand the effect of PM on the patients' quality of life. Especially, CPM for patients with already diagnosed breast cancer has not been evaluated in most studies. In this study, we aimed to analyze the effect of BPM and CPM for patients with high risk for new or additional breast cancer on satisfaction and quality of life using the BREAST-Q questionnaire.

\section{Methods}

\section{Patients}

Between 2014 and 2016 patients at high-risk for breast cancer who sought for bilateral postmastectomy breast reconstruction were offered to participate in this study. Inclusion was restricted to patients undergoing bilateral skin-sparing mastectomy with direct implant- or expander-based reconstruction. Patients were excluded if they declined participation or if they have already been included in another surgical study. BPM in patients with already diagnosed breast cancer is further summarized under the term CPM. Before and 1 year after the PM, the participating women were asked to complete the BREAST-Q questionnaire.

Patient- and procedure-related information was collected from the patient's medical records and archived in a database.

\section{Surgery}

In all of the patients bilateral skin-sparing or nipple-sparing mastectomy was performed with immediate implant or expander reconstruction in a submuscular position. We used anatomical (i.e., 312, 322, 323) textured implants from Mentor (Mentor Worldwide LLC, Irvine, CA, USA). Mastectomy and reconstruction were done in one procedure by the senior author. We used acellular dermal matrix or a mesh in order to add an extra layer of tissue between the expander/implant and the skin and to fix the implant position as described in the literature $[13,14]$. As acellular dermal matrix we used Strattice (Allergan, Dublin, Ireland) and as mesh we applied TiLOOP ${ }^{\circledR}$ Bra (pfm medical, Cologne, Germany) or TIGR Matrix (Novus Scientific, Uppsala, Sweden). For women with a larger breast size a wise pattern mastectomy with a tissue expander using a dual plane of vascularized tissue coverage was used as published by other authors [15, 16]. Nipple-sparing mastectomy, nipple reconstruction, and tattooing were offered to the patients after a detailed explanation of the risks and benefits.

\section{BREAST-Q}

The BREAST-Q questionnaire is a patient-related outcome instrument that includes various modules regarding satisfaction and quality of life after breast-related surgery as described in by Pusic et al. [12]. We used the reconstruction module provided in German by the Mapi Research Trust (https://mapi-trust.org/). Answers were provided according to a 4 - or 5-point ordinal scale. The results of the individual questions were summarized in a domainspecific score ranging from 0 (worst) to 100 (best) according to a provided protocol. Patients were invited for a follow-up examination at the office. A hard-copy of the questionnaire was handed out and a standardized photo was taken to document postoperative results.

Statistical analysis was performed using R (http://www.cran.rproject.org). Mean scores were compared using a paired $t$ test. $p<$ 0.05 was considered statistically significant.

\section{Results}

In total, 49 patients were asked to participate. One patient declined participation. We included 48 patients undergoing bilateral skin-sparing mastectomy in this study. All of the patients were at high risk for breast cancer (breast cancer-promoting genetic mutation or family history). BRCA1 and BRCA2 mutations were detected in 39 out of $48(81.3 \%)$ patients. In total, $29(60.4 \%)$ patients underwent surgery due to already diagnosed breast cancer. All breast cancer patients in this study were treated with additional CPM. Most cancer patients (44.8\%) presented with T2 tumors. A 2-stage reconstruction with intermediate expander implantation was conducted in 19 (39.6\%) patients. Synthetic mesh and acellular matrices were applied in $29(60.4 \%)$ and $15(31.3 \%)$ patients, respectively. In total, $30(62.5 \%)$ patients opted for a nipplesparing mastectomy. Of the remaining 18 patients, 9 received immediate reconstruction of the nipples with a modification of a CV flap. Of these, 3 patients decided to have a tattoo of the reconstructed nipple and an areola tattoo. One patient decided to have a tattoo of the nippleareola complex without reconstruction of the nipple. The remaining 7 patients refused to have nipple reconstruction or tattoo. Perioperative complications occurred in 7 
out of 96 (7.2\%) breasts. Further details on the patients' characteristics, tumor characteristics, and surgical procedures are shown in Table 1.

All of the patients completed the BREAST-Q questionnaire before and 1 year after surgery. The results for the main domains are shown in Table 2. As indicated, the domain "satisfaction with breast" appeared to be slightly, but not significantly, improved after surgery, with a mean increase of 3.08 points $(p=0.319)$. Interestingly, the domain "psychosocial well-being" was significantly improved from a mean score of 74.98 preoperatively to a postoperative score of $81.56(p=0.021)$. In contrast, the domain "physical well-being/chest and upper body" dropped an average of -8.38 points to a postoperative score $74.96(p<0.001)$. The noncomparable postoperative domains "satisfaction with result," "with nipples," "with information," "with surgeon," and "with medical team" congruently achieved high results ranging from 61.10 points for "satisfaction with nipples" to 94.11 points for "satisfaction with surgeon."

Comparing patients undergoing BPM with patients already diagnosed with breast cancer, we did not observe significant differences regarding the different domains (data not shown). Analyzing pre- and postoperative results in the separate groups, we found a significant decrease in the domain "physical well-being/chest and upper body" for oncological patients from 83.41 to 73.66 $(p=0.001)$. This difference could not be observed in the PBM group. Table 3 displays the exact results.

Additionally, we thoroughly investigated how the postoperative domains depended on the preoperative results regarding the domain "satisfaction with breast." Table 4 shows the results. The entire cohort was divided into 3 subgroups depending on the score of the preoperative domain "satisfaction with breast." Interestingly, we observed a clear difference regarding the change in values between pre- and postoperative assessments. The third group with the lowest preoperative satisfaction showed the greatest increase after the operation (50.31 vs. 67.25 , $p<0.001)$. On the contrary, the patients with the highest preoperative values experienced the strongest decrease in satisfaction (91.60 vs. 75.27; $p=0.012$ ). Figures 1 and 2 show exemplary pre- and postoperative images of patients in the respective group. Similarly, we noticed a significant increase in scores regarding sexual well-being (48.06-66.39, $p=0.03$ ) and unchanged scores regarding physical well-being/chest and upper body (66.50 vs. 67.07, $p=0.729$ ) in the group of patients with the lowest preoperative satisfaction with the breast. In contrast, patients with the highest preoperative satisfaction reported unchanged scores regarding sexual well-being (82.64 vs. 83.36, $p=0.843$ ) and decreased scores on physical wellbeing ( 100.00 vs. $82.93, p=0.002$ ). Of note, regarding absolute scores all postoperative scores in the high-satisfac-
Table 1. Patient characteristics

\begin{tabular}{|c|c|}
\hline Age, years & $39(24-55)$ \\
\hline BMI & $24(17-31)$ \\
\hline History of diabetes & $0(0.0)$ \\
\hline Active smoker & $1(2.1)$ \\
\hline History of radiotherapy & $1(2.1)$ \\
\hline \multicolumn{2}{|l|}{ Surgery } \\
\hline Prohylactic (BPM) & $19(39.6)$ \\
\hline Breast cancer + CPM & $29(60.4)$ \\
\hline Bilateral skin-sparing mastectomy & $48(100)$ \\
\hline $\begin{array}{l}\text { Uni- or bilateral nipple-sparing } \\
\text { mastectomy }\end{array}$ & $30(62.5)$ \\
\hline Expander/implant reconstruction & $19(39.6)$ \\
\hline Direct implant reconstruction & $29(60.4)$ \\
\hline Axillary lymph node dissection & $2(4.2)$ \\
\hline Acellular matrix & $15(31.3)$ \\
\hline Synthetic mesh & $29(60.4)$ \\
\hline $\begin{array}{l}\text { Vascularized dermal-subcutaneous } \\
\text { pedicle }\end{array}$ & $2(4.2)$ \\
\hline Complete muscular cover & $2(4.2)$ \\
\hline Hospital stay, days & $12(7-24)$ \\
\hline \multicolumn{2}{|l|}{ Resected breast tissue } \\
\hline Right & $242.5(74.8-766.0)$ \\
\hline Left & $250.0(70.9-792.0)$ \\
\hline Expander volume, $\mathrm{mL}$ & $270.0(140.0-460.0)$ \\
\hline Implant size, $\mathrm{mL}$ & $295.0(175.0-475.0)$ \\
\hline \multicolumn{2}{|l|}{ Tumor } \\
\hline \multicolumn{2}{|l|}{ Size } \\
\hline pTis & $2(4.2)$ \\
\hline cT1 & $11(22.9)$ \\
\hline cT2 & $12(25.0)$ \\
\hline cT3 & $3(6.3)$ \\
\hline \multicolumn{2}{|l|}{ Adjuvant therapy } \\
\hline Chemotherapy & $8(16.7)$ \\
\hline Neoadjuvant chemotherapy & $18(37.5)$ \\
\hline Radiotherapy & $5(10.4)$ \\
\hline Complications & $12(25.0)$ \\
\hline \multicolumn{2}{|l|}{ Major } \\
\hline Hematoma with revision & $5(10.4)$ \\
\hline Implant loss & $0(0.0)$ \\
\hline Implant rotation & $1(2.1)$ \\
\hline Wound healing disorder with revision & $1(2.1)$ \\
\hline \multicolumn{2}{|l|}{ Minor } \\
\hline Wound healing disorder & $1(2.1)$ \\
\hline
\end{tabular}

Values are presented as medians (range) or numbers (\%).

tion group were still higher compared to those of the lowsatisfaction cohort. The results are shown in Table 4.

There were no recurrences during the study period.

\section{Discussion}

In this study we were able to show that patients undergoing BPM or CPM with 1- or 2-stage reconstruction experience significantly better BREAST-Q scores, regarding psychosocial well-being, 1 year after surgery. However, reduced scores were detected regarding physical 
Table 2. Pre- and postoperative BREAST-Q scores for all of the included patients

\begin{tabular}{|c|c|c|c|c|}
\hline Domain $(n=49$, range $0-100)$ & Preoperative score & Postoperative score & $\begin{array}{l}\text { Mean difference } \\
(95 \% \text { CI })\end{array}$ & $p$ value \\
\hline Satisfaction with breast & $69.54 \pm 18.31$ & $72.63 \pm 14.99$ & $3.08(9.24$ to -3.07$)$ & 0.319 \\
\hline Psychosocial well-being & $74.98 \pm 18.20$ & $81.56 \pm 20.48$ & $6.58(12.12$ to 1.05$)$ & 0.021 \\
\hline Physical well-being/chest and upper body & $83.33 \pm 14.20$ & $74.96 \pm 14.12$ & $-8.38(-4.22$ to -12.53$)$ & $<0.001$ \\
\hline Sexual well-being & $61.53 \pm 15.35$ & $69.19 \pm 21.44$ & $6.89(13.18$ to 0.6$)$ & 0.033 \\
\hline Satisfaction with result & & $85.44 \pm 16.46$ & & \\
\hline Satisfaction with nipples & & $61.10 \pm 36.66$ & & \\
\hline Satisfaction with information & & $81.26 \pm 20.22$ & & \\
\hline Satisfaction with surgeon & & $94.11 \pm 11.46$ & & \\
\hline Satisfaction with medical team & & $87.94 \pm 18.98$ & & \\
\hline
\end{tabular}

Values are presented as means \pm SD unless otherwise stated. Bold numbers represent statistically significant values.

Table 3. Change in BREAST-Q results for prophylactic and oncological patients after surgery

\begin{tabular}{|c|c|c|c|c|}
\hline Domain & Preoperative results & Postoperative results & $\begin{array}{l}\text { Mean difference } \\
(95 \% \text { CI })\end{array}$ & $p$ value \\
\hline \multicolumn{5}{|l|}{ Oncological $(n=28)$} \\
\hline Satisfaction with breast & $73.41 \pm 18.42$ & $72.52 \pm 15.68$ & $-0.90(7.50$ to -9.30$)$ & 0.828 \\
\hline Psychosocial well-being & $74.79 \pm 18.24$ & $80.38 \pm 19.42$ & $5.59(12.60$ to -1.42$)$ & 0.113 \\
\hline Physical well-being/chest and upper body & $83.41 \pm 13.14$ & $73.66 \pm 12.45$ & $-9.76(-4.10$ to -15.42$)$ & 0.001 \\
\hline Sexual well-being & $59.65 \pm 15.40$ & $67.71 \pm 18.82$ & $6.62(13.69$ to -0.45$)$ & 0.065 \\
\hline \multicolumn{5}{|l|}{ Prophylactic $(n=21)$} \\
\hline Satisfaction with breast & $63.63 \pm 16.94$ & $72.79 \pm 14.30$ & $9.16(18.31$ to 0.01$)$ & 0.050 \\
\hline Psychosocial well-being & $75.26 \pm 18.64$ & $83.37 \pm 22.43$ & $8.11(17.84$ to -1.62$)$ & 0.097 \\
\hline Physical well-being/chest and upper body & $83.21 \pm 16.06$ & $76.95 \pm 16.52$ & $-6.26(0.28$ to -12.80$)$ & 0.060 \\
\hline Sexual well-being & $64.11 \pm 15.30$ & $71.37 \pm 25.21$ & $7.26(19.40$ to -4.88$)$ & 0.227 \\
\hline
\end{tabular}

Values are presented as means \pm SD unless otherwise stated. Bold numbers represent statistically significant values.

Table 4. Pre- and postoperative BREAST-Q results depending on preoperative values for the domain "satisfaction with breast"

\begin{tabular}{lcccc}
\hline & Preoperative result & Postoperative result & $\begin{array}{l}\text { Mean difference } \\
(95 \% \text { CI) }\end{array}$ \\
\hline Upper third $(n=16)$ & & & \\
$\quad$ Satisfaction with breast & $91.60 \pm 10.65$ & $75.27 \pm 18.07$ & $-16.33(-4.12$ to -28.54$)$ & $\mathbf{0 . 0 1 2}$ \\
Psychosocial well-being & $96.92 \pm 4.05$ & $95.46 \pm 7.73$ & $-1.46(3.26$ to -6.18$)$ & 0.510 \\
Physical well-being/chest and upper body & $100.00 \pm 0.00$ & $82.93 \pm 16.15$ & $-17.07(-7.67$ to -26.47$)$ & $\mathbf{0 . 0 0 2}$ \\
Sexual well-being & $82.64 \pm 12.27$ & $83.36 \pm 12.44$ & $0.73(8.69$ to -7.23$)$ & 0.843 \\
Lower third $(n=16)$ & & & $16.94(23.94$ to 9.93$) \quad<\mathbf{0 . 0 0 1}$ \\
Satisfaction with breast & $50.31 \pm 5.68$ & $67.25 \pm 12.60$ & $14.53(30.18$ to -1.12$)$ & 0.066 \\
Psychosocial well-being & $53.60 \pm 5.54$ & $68.13 \pm 25.16$ & $0.57(4.09$ to -2.94$)$ & 0.729 \\
Physical well-being/chest and upper body & $66.50 \pm 8.71$ & $67.07 \pm 11.91$ & $18.33(29.41$ to 7.26$)$ & $\mathbf{0 . 0 0 3}$
\end{tabular}

Values are presented as means \pm SD unless otherwise stated. Bold numbers represent statistically significant values. 

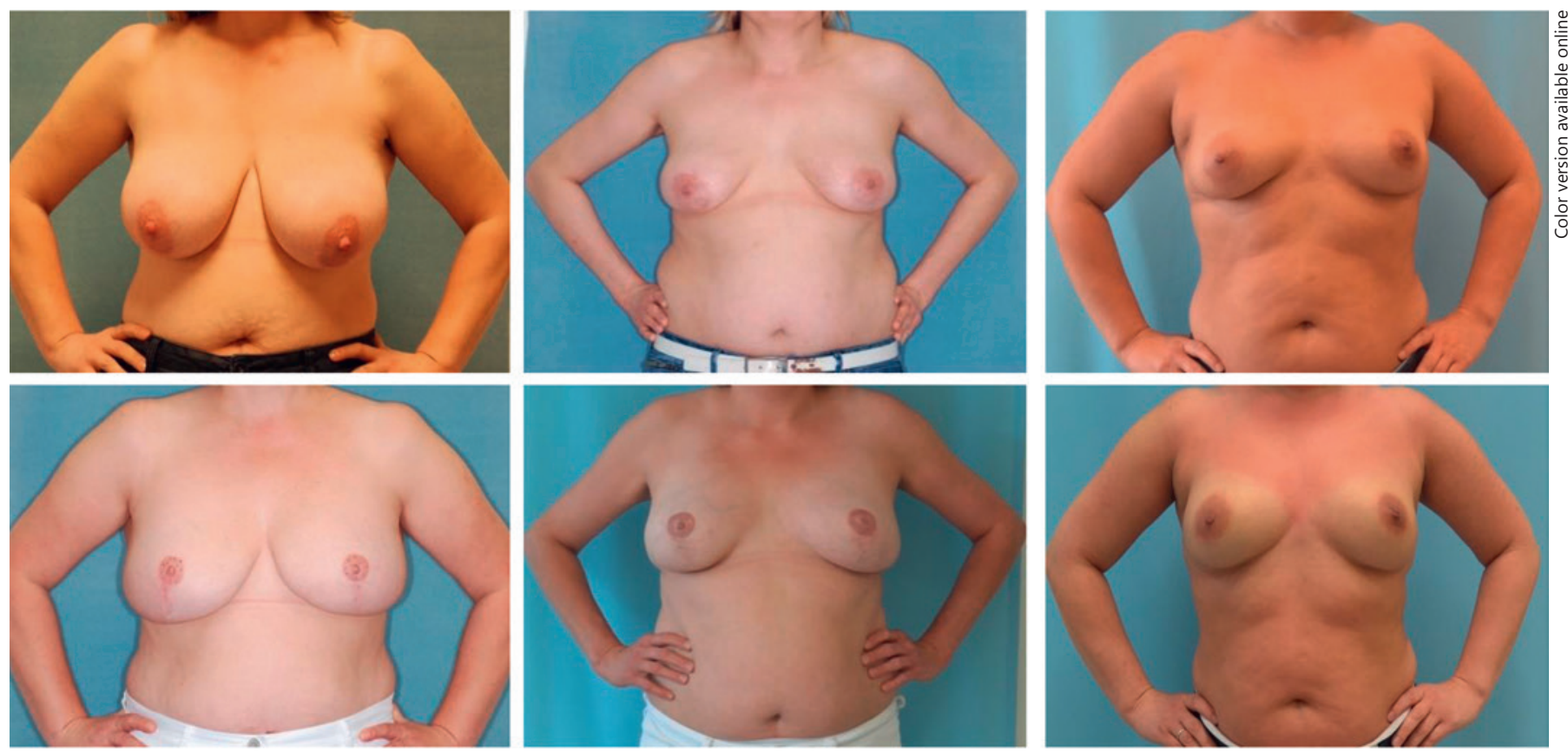

Scores Breast-Q domain ,Satisfaction with breast ${ }^{\prime}$

\begin{tabular}{|l|c|c|c|}
\hline & Patient 1 & Patient 2 & Patient 3 \\
\hline Preoperatively & 43 & 48 & 53 \\
\hline Postoperatively & 81 & 75 & 85 \\
\hline
\end{tabular}

Fig. 1. Exemplary pre- (top) and postoperative (bottom) pictures of 3 patients with low scores in the preoperative domain "satisfaction with breast." The table shows the exact scores of each patient.

well-being/chest and upper body 1 year after surgery. We showed that patients with a high satisfaction with their breast in the preoperative questionnaire have a higher risk of reduced scores postoperatively. On the contrary, patients who are less satisfied with the preoperative examination are more likely to have improved scores postoperatively.

As mentioned in the Introduction, decision making regarding PM is a complex process. On the one hand we are aware of the strong breast cancer risk reduction of $\mathrm{PM}$ for women at high risk [1]. On the other hand these procedures are complex surgeries possibly associated with severe perioperative complications and have a potential impact on the body image and quality of life of the patients [2-8]. Healthy patients with an elevated breast cancer risk have the medically accepted choice to decline surgery and undergo screening $[17,18]$. Additionally, most patients eligible for prophylactic surgery are young [19]. The effects on these patients' body image and quality of life might be different to those on older patients or patients already diagnosed with breast cancer [20]. All of these concerns have to be addressed during individual counseling. The advantages of PM have to be weighed against the above mentioned possible negative effects. Therefore, more data is required to better select and counsel patients considering individual factors.

In our cohort, we saw major complications in 7 out of $96(7.2 \%)$ breasts. These rates appear to be in line with those already published in various studies on BPM, which vary extremely depending on the selected surgical approach and follow-up $[8,21,22]$. All our complications could be treated without removing the implant. Our patients stayed comparably long in our hospital. This can be explained by our quite conservative postoperative management. Our patients stay in hospital until the drains are removed. We remove the drains if there is $<30 \mathrm{~mL}$ of secretion per day.

From previous studies on PM we know that $50-100 \%$ of patients are satisfied with their result and $80-90 \%$ of patients are satisfied with their decision to have a PM performed [3-6, 9]. Regarding the patient's body image after PM, previous studies have reported different results ranging from increased to decreased scores in postoperative questionnaires $[4,5,7]$. A major limitation on a comprehensive comparison with most studies is the diversity of the applied questionnaires. There was a lack of specific 

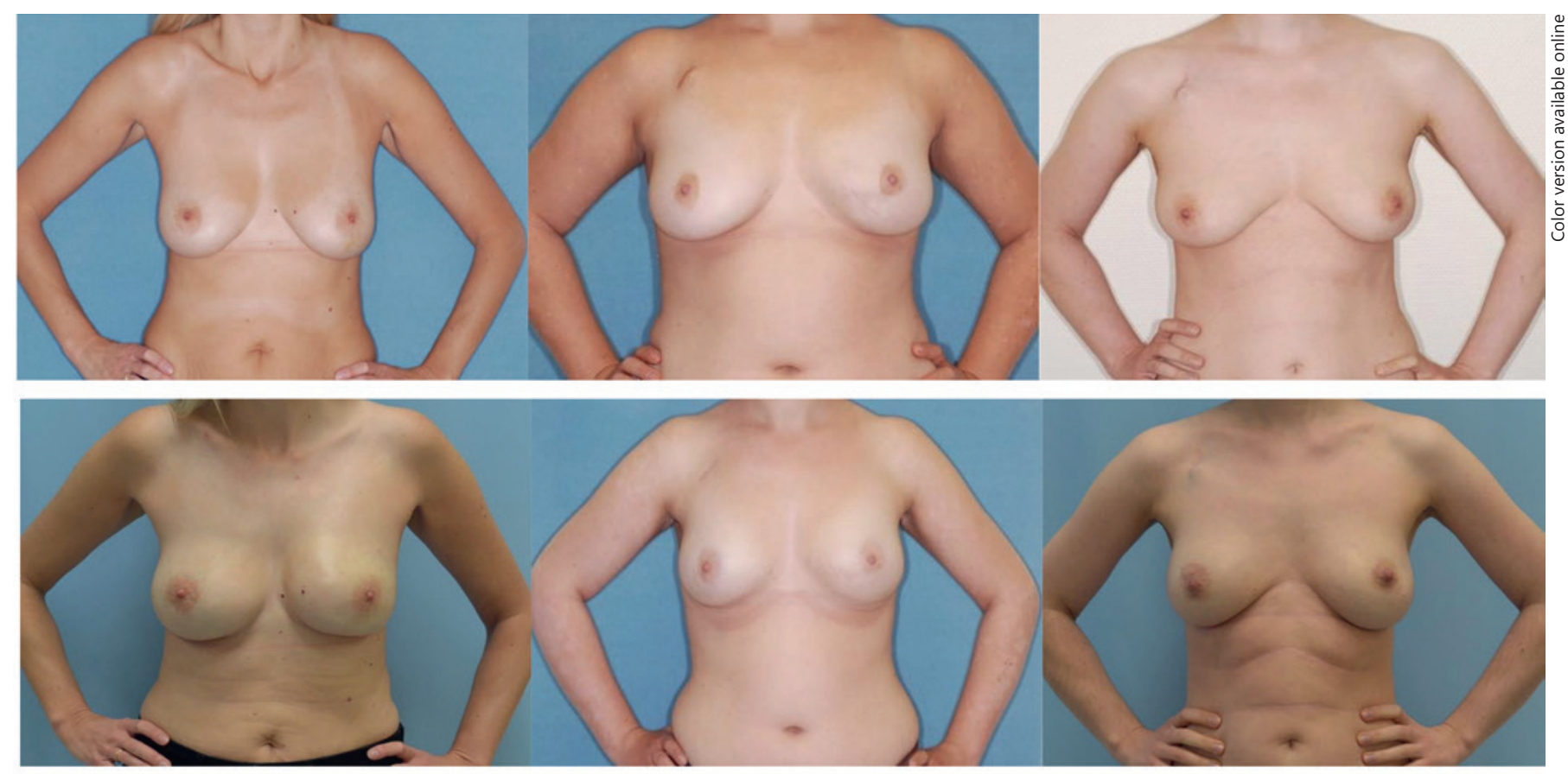

Scores Breast-Q domain ,Satisfaction with breast'

\begin{tabular}{|l|c|c|c|}
\hline & Patient 4 & Patient 5 & Patient 6 \\
\hline Preoperatively & 100 & 100 & 100 \\
\hline Postoperatively & 100 & 78 & 65 \\
\hline
\end{tabular}

Fig. 2. Exemplary pre- (top) and postoperative (bottom) pictures of 3 patients with high scores in the preoperative domain "satisfaction with breast." The table shows the exact scores of each patient.

questionnaires for breast reconstruction before the BREAST-Q questionnaire had been introduced [12]. Focusing on one comprehensive questionnaire in future studies will definitely improve the comparability of the results from different studies. Also, after having analyzed the wide heterogeneity of the quality-of-life assessment tools used after PM, Razdan et al. [9] recommended in their review the use of the BREAST-Q questionnaire in future studies. Therefore, the use of this standardized breast-specific questionnaire on patients undergoing solely implant-based BPM and CPM makes our study unique.

In our study, we noted high scores (ranging from 81 to 88) regarding the postoperative items ("satisfaction with result," "with information," "with surgeon," and "with medical team") except for the domain "satisfaction with nipples" (score 61.1). Interestingly, comparing pre- and postoperative domains we also observed a slightly, but not significantly, improved score for "sexual well-being" and a significantly improved score for "psychosocial wellbeing." The "physical well-being/chest and upper body" slightly decreased after surgery. Comparing our results to the only large study on PM which also used the BREAST-
Q questionnaire, we noticed similar values and trends except for the item "satisfaction with breast" [8]. Interestingly, the baseline score in the study by McCarthy et al. [8] was apparently lower (i.e., 59.3) than ours (i.e., 71.98). The baseline characteristics of the included patients were quite similar; McCarthy et al. [8] also included women treated with autologous breast reconstruction. This, however, should not affect preoperative BREAST-Q scores. In contrast to McCarthy et al. [8], we also included patients already diagnosed with breast cancer undergoing therapeutic mastectomy and CPM. Interestingly, we did not see any significant differences regarding the postoperative items related to the surgical procedure and result between patients treated with BPM or CPM. Patients already diagnosed with cancer appeared to have a higher preoperative "satisfaction with breast" (i.e., 74.54 vs. 68.57) as well as a stronger decrease in the domain "physical well-being/chest and upper body" (-8.86 vs. -2.76$)$. There are no comparable studies on the difference between BPM and CPM using the BREAST-Q questionnaire with which or results can be compared. In the study by Altschuler et al. [23] using open-ended questions to assess satisfaction with the surgery, the percentage of pa- 
tients with negative or disparate responses was twice as high for BPM (52\%) compared to CPM (26\%). Other groups reported good quality-of-life results after CPM which are comparable to those of BPM from other studies $[24,25]$.

To our knowledge, there is only one small study with 10 women correlating the preoperative satisfaction with the breast with postoperative outcomes [26]. According to our results, the information on preoperative satisfaction does have a significant influence on the postoperative results. Women highly satisfied with their breast preoperatively (domain score 91.60) experienced a significant decrease regarding the domains "satisfaction with breast" $(-16.33, p=0.012)$ and physical well-being/chest and upper body $(-17.07, p=0.002)$ and no change regarding "psychosocial" and "sexual well-being." In contrast, in patients with a low satisfaction with their breast (domain score 50.31) we noticed a significant improvement in the domains "satisfaction with breast" (i.e., 16.94, $p=$ 0.001 ) and "sexual well-being" (i.e., 18.33, $p=0.003$ ). These results might be of particular importance when counseling patients before PM. Especially, patients with a low preoperative satisfaction with their breast have significantly higher chances of experiencing substantial improvements in their postoperative quality of life. Highly satisfied patients will more likely be less satisfied after surgery. However, in our study, they still reported good to excellent scores for body image and quality of life. In future studies it would be very interesting to look deeper into the reasons for a lower preoperative satisfaction with the breasts and the impact on postoperative results.

Due to the small groups, we did not test for the impact of nipple-sparing mastectomy on BREAST-Q outcomes $[10,11]$.

Finally, some limitations of this study have to be mentioned. With 48 included patients, we did not reach significance in some analyses. However, we report an average sample size compared to similar studies and our prospective assessment supports the reliability of our results. We report results from surgeries and counseling by one highly experienced surgeon. This led to good and reliable results in this study. It has to be kept in mind when transferring our results into clinical practice that counseling, a physician-initiated discussion, complications, and postoperative results are surgeon dependent and they are associated with postoperative satisfaction [3]. In this study we reported a 1-year follow-up. The quality of life and complications might change over time. Therefore, further follow-up visits are planned to assess late effects.

Experts agree that patient counseling and patient selection before PM are the key to a high postoperative satisfaction and quality of life. Using the BREAST-Q questionnaire for preoperative assessment could improve the individual counseling and set reasonable expectations regarding the postoperative result.

\section{Statement of Ethics}

All of the patients provided informed consent. This study was approved by the institutional Ethics Committee.

\section{Disclosure Statement}

U.H.-B. participated in the ESSBR study. Patients from the EBSSR study were not included in this analysis. All of other authors declare no conflict of interests.

\section{Funding Sources}

There are no funding sources to declare.

\section{Author Contributions}

All of the authors materially participated in this research, were involved in the preparation of this article, and approved the final version. U.H.-B., R.K., and C.W. were involved in the study conception and design. U.H.-B., A.P., J.E., and C.W. were involved in acquisition of data. R.K., U.H.-B., and C.W. were involved in analysis and interpretation of the data. R.K. and U.H.-B. wrote the initial draft of this paper. R.K., U.H.-B., C.W., A.P., F.K., E.K., and J.E. were involved in critical revision of this work.

\section{References}

1 Li X, You R, Wang X, Liu C, Xu Z, Zhou J, et al. Effectiveness of Prophylactic Surgeries in BRCA1 or BRCA2 Mutation Carriers: A Meta-analysis and Systematic Review. Clin Cancer Res. 2016 Aug;22(15):3971-81.

2 Antoniou A, Pharoah PD, Narod S, Risch HA, Eyfjord JE, Hopper JL, et al. Average risks of breast and ovarian cancer associated with BRCA1 or BRCA2 mutations detected in case Series unselected for family history: a combined analysis of 22 studies. Am J Hum Genet. 2003 May;72(5):111730.
3 Borgen PI, Hill AD, Tran KN, Van Zee KJ, Massie MJ, Payne D, et al. Patient regrets after bilateral prophylactic mastectomy. Ann Surg Oncol. 1998 Oct-Nov;5(7):603-6.

4 Brandberg Y, Sandelin K, Erikson S, Jurell G, Liljegren A, Lindblom A, et al. Psychological reactions, quality of life, and body image after bilateral prophylactic mastectomy in women at high risk for breast cancer: a prospective 1-year follow-up study. J Clin Oncol. 2008 Aug;26(24):3943-9.

5 Frost MH, Schaid DJ, Sellers TA, Slezak JM, Arnold PG, Woods JE, et al. Long-term satis- faction and psychological and social function following bilateral prophylactic mastectomy. JAMA. 2000 Jul;284(3):319-24.

6 Geiger AM, Nekhlyudov L, Herrinton LJ, Rolnick SJ, Greene SM, West CN, et al. Quality of life after bilateral prophylactic mastectomy. Ann Surg Oncol. 2007 Feb;14(2):686-94.

7 Gopie JP, Mureau MA, Seynaeve C, Ter Kuile MM, Menke-Pluymers MB, Timman R, et al. Body image issues after bilateral prophylactic mastectomy with breast reconstruction in healthy women at risk for hereditary breast cancer. Fam Cancer. 2013 Sep;12(3):479-87. 
8 McCarthy CM, Hamill JB, Kim HM, Qi J, Wilkins E, Pusic AL. Impact of Bilateral Prophylactic Mastectomy and Immediate Reconstruction on Health-Related Quality of Life in Women at High Risk for Breast Carcinoma: Results of the Mastectomy Reconstruction Outcomes Consortium Study. Ann Surg Oncol. 2017 Sep;24(9):2502-8.

9 Razdan SN, Patel V, Jewell S, McCarthy CM. Quality of life among patients after bilateral prophylactic mastectomy: a systematic review of patient-reported outcomes. Qual Life Res. 2016 Jun;25(6):1409-21.

10 Romanoff A, Zabor EC, Stempel M, Sacchini V, Pusic A, Morrow M. A Comparison of Patient-Reported Outcomes After Nipple-Sparing Mastectomy and Conventional Mastectomy with Reconstruction. Ann Surg Oncol. 2018 Oct;25(10):2909-16.

11 Casella D, Di Taranto G, Marcasciano M, Sordi S, Kothari A, Kovacs T, et al. Nipple-sparing bilateral prophylactic mastectomy and immediate reconstruction with TiLoop ${ }^{\circledR}$ Bra mesh in BRCA1/2 mutation carriers: A prospective study of long-term and patient reported outcomes using the BREAST-Q. Breast. 2018 Jun;39:8-13.

12 Pusic AL, Klassen AF, Scott AM, Klok JA, Cordeiro PG, Cano SJ. Development of a new patient-reported outcome measure for breast surgery: the BREAST-Q. Plast Reconstr Surg. 2009 Aug; 124(2):345-53.

13 Dieterich M, Paepke S, Zwiefel K, Dieterich H, Blohmer J, Faridi A, et al. Implant-based breast reconstruction using a titanium-coated polypropylene mesh (TiLOOP Bra): a multicenter study of 231 cases. Plast Reconstr Surg. 2013 Jul;132(1):8e-19e.
14 Salzberg CA, Dunavant C, Nocera N. Immediate breast reconstruction using porcine acellular dermal matrix (Strattice ${ }^{\mathrm{TM}}$ ): longterm outcomes and complications. J Plast Reconstr Aesthet Surg. 2013 Mar;66(3):323-8.

15 Losken A, Collins BA, Carlson GW. Dualplane prosthetic reconstruction using the modified wise pattern mastectomy and fasciocutaneous flap in women with macromastia. Plast Reconstr Surg. 2010 Sep;126(3):731-

16 Derderian CA, Karp NS, Choi M. Wise-pattern breast reconstruction: modification using AlloDerm and a vascularized dermal-subcutaneous pedicle. Ann Plast Surg. 2009 May; 62(5):528-32.

17 Ludwig KK, Neuner J, Butler A, Geurts JL, Kong AL. Risk reduction and survival benefit of prophylactic surgery in BRCA mutation carriers, a systematic review. Am J Surg. 2016 Oct;212(4):660-9.

18 Llort G, Chirivella I, Morales R, Serrano R, Sanchez AB, Teulé A, et al.; SEOM Hereditary Cancer Working Group. SEOM clinical guidelines in Hereditary Breast and ovarian cancer. Clin Transl Oncol. 2015 Dec;17(12): 956-61.

19 Skytte AB, Gerdes AM, Andersen MK, Sunde $\mathrm{L}$, Brøndum-Nielsen $\mathrm{K}$, Waldstrøm $\mathrm{M}$, et al. Risk-reducing mastectomy and salpingo-oophorectomy in unaffected BRCA mutation carriers: uptake and timing. Clin Genet. 2010 Apr;77(4):342-9.
20 Tiggemann M, Lynch JE. Body image across the life span in adult women: the role of selfobjectification. Dev Psychol. 2001 Mar;37(2): 243-53.

21 Carbine NE, Lostumbo L, Wallace J, Ko H. Risk-reducing mastectomy for the prevention of primary breast cancer. Cochrane Database Syst Rev. 2018 Apr 5;4:CD002748.

22 Spear SL, Schwarz KA, Venturi ML, Barbosa T, Al-Attar A. Prophylactic mastectomy and reconstruction: clinical outcomes and patient satisfaction. Plast Reconstr Surg. 2008 Jul; 122(1):1-9.

23 Altschuler A, Nekhlyudov L, Rolnick SJ, Greene SM, Elmore JG, West CN, et al. Positive, negative, and disparate-women's differing long-term psychosocial experiences of bilateral or contralateral prophylactic mastectomy. Breast J. 2008 Jan-Feb;14(1):25-32.

24 Frost MH, Slezak JM, Tran NV, Williams CI, Johnson JL, Woods JE, et al. Satisfaction after contralateral prophylactic mastectomy: the significance of mastectomy type, reconstructive complications, and body appearance. J Clin Oncol. 2005 Nov;23(31):7849-56.

25 Geiger AM, West CN, Nekhlyudov L, Herrinton LJ, Liu IL, Altschuler A, et al. Contentment with quality of life among breast cancer survivors with and without contralateral prophylactic mastectomy. J Clin Oncol. 2006 Mar;24(9):1350-6.

26 Lloyd SM, Watson M, Oaker G, Sacks N, Querci della Rovere U, Gui G. Understanding the experience of prophylactic bilateral mastectomy: a qualitative study of ten women. Psychooncology. 2000 Nov-Dec;9(6):473-85. 